\title{
Noise Adaptive FCM algorithm for Segmentation of MRI Brain Images
}

\author{
Harmanpreet Singh* and Ramandeep Kaur \\ Modern Institute of Engineering and Technology, Mohri, Haryana, India \\ Received 01 May 2018, Accepted 01 July 2018, Available online 02 July 2018, Vol.8, No.4 (July/Aug 2018)
}

\begin{abstract}
Image segmentation is an important task in many image processing applications. Fuzzy C means algorithm has been widely used for the segmentation. There are many versions of the traditional FCM algorithm which uses the local spatial information to increase the robustness of this algorithm in presence of noise, but all these algorithms do not successfully segment the images contaminated by heavy noise. In order to solve this problem, non-local spatial information present in the image is utilized. The filtering parameter ' $h$ ' in the non-local information is a crucial parameter which needs to be appropriately determined, irrespective of using a single constant value of ' $h$ '; we can determine its value using the standard deviation of noise present in the image. The adaptive non-local information determined is termed as noise adaptive non-local spatial information. This adaptive non-local information is used in the FCM algorithm for the segmentation of MRI images. In this paper Noise adaptive FCM algorithm (NAFCM) using adaptive non-local information is proposed. Therefore the proposed algorithm utilizes adaptive non-local information making it robust in presence of noise as well as preserving the details present in the image. The efficiency of the proposed algorithm is demonstrated by validation studies on synthetic as well as simulated brain MRI images. The results of the proposed algorithm show that the proposed algorithm is robust to noise and as compared to other state of the art algorithms.
\end{abstract}

Keywords: Image segmentation, Fuzzy c-means, Magnetic Resonance image, non-local spatial, Rician noise

\section{Introduction}

Image segmentation is used in many image processing applications; which include medical researches and clinical applications. The motive of image segmentation is to partition the image into sub-regions depending upon some criteria. Image segmentation algorithms are mainly classified into four categories: Edge detection, region extraction, thresholding and clustering algorithms. In this paper, clustering based algorithm is used for the segmentation purpose. Clustering algorithm segments an image by grouping similar data points in the feature space into the same cluster. FCM is commonly used clustering algorithm for image segmentation. FCM was introduced by Dunn and promoted later by Bezdek. The motive of this algorithm is to divide the image into clusters of similar data points by minimizing a cost function with respect to a distance metric, which depends upon the distance between the pixels and the cluster centers in the feature space. FCM algorithm only considers the intensity information and do not take into account the spatial information present in the image into consideration, therefore it is highly sensitive to noise.

*Corresponding author's ORCID ID: 0000-0001-5499-9348 DOI: https://doi.org/10.14741/ijcet/v.8.4.4
There are many clustering algorithms discussed in the literature for segmentation. Ahmed et al. proposed an algorithm named FCM_S by incorporating a neighbor regularization term into the objective function of the FCM. This algorithm improves the noise sensitivity but the computational time is increased. Chen and Zhang proposed two variants named FCM_S1 and FCM_S2, which use the mean filtered and median filtered image respectively to replace the neighborhood term in FCM_S and these filtered images are computed in advance thus decreasing the computational time.

Szilgyi et al proposed EnFCM, which uses the grey level histogram for performing clustering and the numbers of grey levels are less in comparison with the number of pixels in the image, thus decreasing the time complexity. To further reduce the computational time Cai et al. proposed FGFCM which incorporates both the local spatial and the local grey level relationship into the FCM algorithm, thereby decreasing the noise sensitivity and also reduces the time complexity. All the above mentioned algorithms use the local spatial information for segmentation. In case of MRI images corrupted by high amount of noise, the efficiency of all these algorithms decreases; which is the major drawback of all these algorithms. Zexuan et.al 
proposed WIPFCM, which uses the image patches instead of pixels in FCM algorithm to improve the robustness. Motivated by patch based methods Zaixin et.al proposed NWFCM, which uses both the patch difference and local statistics to introduce a new similarity measure into the objective function of the FCM algorithm, making it to be more efficient than other algorithms.

Zhao, F et al proposed FCM_NLS algorithm which uses the non-local spatial information to increase the robustness in the presence of high noise and the most crucial filtering parameter ' $h$ ' in the non-local information is fixed to a constant value by hit and trial method.

Although all the patch based or non-local information based FCM algorithms are robust to noise , but they fail to segment images in presence of low noise, as the non- local information cannot exploit the accurate information during the assignment of the pixel to a cluster center in case of low noise. Therefore an approach is required, which works well in presence of low as well as high noise.

In(Buades et $a l$ ), for the $\mathrm{i}^{\text {th }}$ pixel, the spatial information derives from a large region of the image rather than a small neighborhood window around it. Therefore, this spatial information is called non-local spatial information. It should be noted that the filtering parameter ' $h$ ' is a very crucial parameter for the computation of non-local spatial information. If parameter ' $h$ ' is too big, then non-local spatial information of the pixel will lose the detail information present in the image, especially the edges in the image. On the other side if ' $h$ ' is too small, the spatial information will be influenced by the image noise. The filtering parameter ' $\mathrm{h}$ ', which is a very crucial parameter during the extraction of non-local information, is taken equal to a single fixed or a constant value.

Since the filtering parameter ' $h$ ' plays an important role in the acquisition of non -local information from the image, it needs to be set very carefully. Instead of using a single value of the filtering parameter ' $h$ ' ,we calculate the filtering parameter from the standard deviation of noise present in the image, therefore making the non-local information adaptive with respect to the amount of noise and the non-local information calculated by using this adaptive filtering parameter ' $h$ ' is termed as noise adaptive non-local spatial information.

In this dissertation work, a Noise adaptive FCM (NAFCM) algorithm is proposed. The similarity measure in the objective function of conventional FCM algorithm is influenced by the adaptive non-local spatial information depending upon the noise present in the image, which increases the robustness of the algorithm in low as well as high noisy environments. The paper is organized as follows: In Section 2, we present the traditional FCM algorithm, In Section 3,we present the proposed algorithm and Experiments are presented in Section 4 and conclusion of the paper is given in section 5 .

\section{FCM Clustering Algorithm}

The FCM algorithm partitions a set of data points $X=\left\{X_{1}\right.$, $\left.\mathrm{x}_{2}, \ldots, \mathrm{x}_{\mathrm{n}}\right\}$ into clusters with $2 \leq \mathrm{c}<\mathrm{n}$ depending on iterative optimization and minimizing the objective function given by:

$J_{F C M}=\sum_{i=1}^{n} \sum_{k=1}^{c} u_{i k}^{m} d^{2}\left(x_{i}, v_{k}\right)$

Subject to constraint

$\sum_{k=1}^{c} u_{i k}=1$

Where $0 \leq u_{i k} \leq 1, \forall i$, and $0<\sum_{i=1}^{n} u_{i k}<n, \forall k$

Where $\mathrm{c}$ is the number of clusters and $\mathrm{n}$ is the no. of data points in the feature space. $x_{i}$ denotes the intensity level of the $i_{\text {th }}$ pixel in the feature space. $d^{2}\left(x_{i}, v_{k}\right)=$ $\left\|x_{i}-v_{k}\right\|^{2}$ is squared Euclidean distance between a feature vector and the clusteroid center $v_{i}$ in the feature space. $u_{i k}^{m} \in[0,1]$ is the degree of pixel $\mathrm{x}_{\mathrm{i}}$ belonging to cluster $\mathrm{k}, \mathrm{m} \in(1, \infty)$ represents degree of fuzziness, and generally taken equal to 2 .

According to the traditional FCM algorithm, the objective function will reach a minimum value by updating the fuzzy membership function and the cluster center as follows:

$$
\begin{aligned}
& u_{i k}=\frac{\left(\left\|x_{i}-v_{k}\right\|\right)^{-2} /(m-1)}{\sum_{j=1}^{c}\left(\left\|x_{i}-v_{j}\right\|\right)^{-2} /(m-1)} \\
& v_{k}=\frac{\sum_{i=1}^{n} u_{i k}^{m} x_{i}}{\sum_{i=1}^{n} u_{i k}^{m}}
\end{aligned}
$$

Equations (3) and (4) of updating membership matrix and the cluster center are derived by taking the first derivative of the objective function with respect to $u$ and $\mathrm{v}$ and letting them equals to zero. The algorithm converges when $\max \left\{U^{(z+1)}-U^{(z)}\right\}<\varepsilon$.

\section{Proposed Algorithm}

All extensions of the standard FCM clustering algorithm can reduce the effect of noise to a certain extent, because these extension methods only incorporate local spatial information into the FCM. However, when the noise level in images is high, the adjacent pixels of a noise point may also contain abnormal features and neighborhood spatial information is not sufficient for the perfect segmentation. Therefore, it becomes necessary to use the non-local spatial information contained in the image for the segmentation purpose. The non-local spatial information finds the pixels having similar configuration as that of the pixel of interest. FCM_NLS, consider non-local spatial information for the segmentation purpose, but it suffers from the following drawback: 
Zhao, F et al's FCM_NLS algorithm, the filtering parameter ' $h$ ' used in the computation of non-local information is fixed manually to a single (constant) value in this algorithm. Therefore, if the value of ' $h$ ' is too big, the similarity measure in the objective function will be influenced by the non-local spatial information, even in case of low percentage of noise, leading to lose of detailed information present in the image, especially for image edges. Conversely if ' $h$ ' is low, the similarity measure will be influenced by the local spatial information in case of high percentage of noise, which will cause the spatial information to be still effected by image noise.

Motivated by FCM_NLS algorithm, a more robust FCM algorithm known as NAFCM is proposed in this Dissertation work, which overcome the above mentioned drawback of the FCM_NLS algorithm by incorporating more robust non-local spatial information into the similarity measure of the FCM algorithm.

\section{A. Noise Adaptive FCM algorithm for Segmentation of MRI Brain Images}

From the description about the standard FCM in previous chapter, the membership value decides the segmentation results and the membership value is determined by the distance metric $d^{2}\left(x_{i}, v_{k}\right)$ given in equation (1). In the proposed NAFCM algorithm, the distance measurement depends upon the non-local spatial information as in the FCM_NL and this weighted distance metric is defined as follows:

$$
d_{f}^{2}\left(x_{i}, v_{k}\right)=d_{n l o}^{2}\left(x_{i}, v_{k}\right)
$$

Where $d_{n l o}^{2}$ is the distance measurement influenced by the non-local spatial information and are calculated using equation (6).

\section{B. Calculation of Non-Local Weighted Distance Metric}

The Non-Local weighted distance metric is obtained by using non-local spatial information and it is calculated by averaging the weight of all the pixels in the given noisy image $I$.

$d_{n l o}^{2}\left(x_{i}, v_{k}\right)=\sum_{x_{r} \in I} w_{n l}\left(x_{r}, x_{i}\right) d^{2}\left(x_{r}, v_{k}\right)$

Where $d^{2}\left(x_{r}, v_{k}\right)$ is the Euclidian distance measurement given in equation (1), $w_{n l}\left(x_{r}, x_{i}\right)$ gives the similarity between the pixel $x_{r}$ and $x_{i}$ satisfying the two necessary conditions $0 \leq w_{n l}\left(x_{r}, x_{i}\right) \leq 1$ and $\sum_{x_{r} \in I} w_{n l}\left(x_{r}, x_{i}\right)=1$. The similarity between the two pixels $x_{r}$ and $x_{i}$ depends upon the similarity between the two intensity grey level vector $v\left(N_{r}\right)$ and $v\left(N_{i}\right)$, where $N_{r}$ and $N_{i}$ denotes a fixed neighborhood square window centered at pixel $r$ and $i$ respectively. This non-local weight function $w_{n l}\left(x_{r}, x_{i}\right)$ is given as follows: $w_{n l}\left(x_{r}, x_{i}\right)=\frac{1}{W\left(x_{i}\right)} H\left(x_{r}, x_{i}\right)$

Where $H\left(x_{r}, x_{i}\right)$ is the exponential similarity measure and $W\left(x_{i}\right)$ is the normalization factor:

$$
\begin{aligned}
& H\left(x_{r}, x_{i}\right)=e^{-\frac{\left\|v\left(N_{r}\right)-v\left(N_{i}\right)\right\|_{2, a}^{2}}{h^{2}}} \\
& W\left(x_{i}\right)=\sum_{x_{r} \in I} e^{-\frac{\left\|v\left(N_{r}\right)-v\left(N_{i}\right)\right\|_{2, a}^{2}}{h^{2}}}
\end{aligned}
$$

Here, $\left\|v\left(N_{r}\right)-v\left(N_{i}\right)\right\|_{2, a}^{2}$ is the Gaussian weighted Euclidian distance and ' $a$ ' denotes the standard deviation of Gaussian kernel . The pixels having similar neighborhood configuration to the center pixel will be having larger weights in the average. Here ' $h$ ' is the filtering parameter controlling the decay of weights as a function of squared Euclidean distance. The search for the similar neighborhood configuration will be restricted to the larger portion of the image known as search window and denoted by $\phi_{i}$ and the neighborhood window is denoted as $N_{i}$. In our experiments, the size of search window is set to $7 \times$ 7 and size of neighborhood window is set to $3 \times 3$.

Note that it is possible to set the value of parameter ' $h$ ' automatically according to the noise level of the corrupted image by using $\mathrm{h}=2 p \sigma$. Where $\sigma$ is the standard deviation of noise present in the image and can be calculated directly from the image (AjaFernandez et al, 2008)and ' $p$ ' is the smoothing parameter, which can be adjusted to provide accurate results. Therefore, the non-local spatial information can be made adaptive by varying the filtering parameter ' $h$ ' in accordance with the standard deviation of noise and the non-local spatial information extracted using this adaptive filtering parameter is called as adaptive non-local information.

\section{Proposed Algorithm (NAFCM)}

Step1: Set values for c, $m$ and $\varepsilon$. Where $m$ is generally taken equal to 2 and $\varepsilon>0$ is set to a very small value.

Step2: Randomly initialize the cluster centroid vector $\mathrm{V}=\left[\mathrm{v}_{1}, \mathrm{v}_{2}, \ldots ., \mathrm{v}_{\mathrm{c}}\right]$.

Step3: Initialize the size of search window $\phi_{i}$ and the neighborhood window size $N_{i}$.

Step4: Calculate the distance metric $d_{f}{ }^{2}$ using equation (5).

Step5: Update the membership matrix $\mathrm{U}=u_{i k}$ using $d_{f}{ }^{2}$. Step6: Update the cluster center $v_{k}$ using $u_{i k}$.

Step7: Repeat Steps 4-6, until the following condition is satisfied:

$\left|V_{\text {new }}-V_{\text {old }}\right|<\varepsilon$.

When the algorithm has converged, pixel $i$ is assigned to class $\mathrm{c}$ with the highest membership.

$\arg _{k}=\arg \left\{\max \left\{u_{i k}\right\}\right\} \quad k=1,2, \ldots \ldots, c$ 
a)
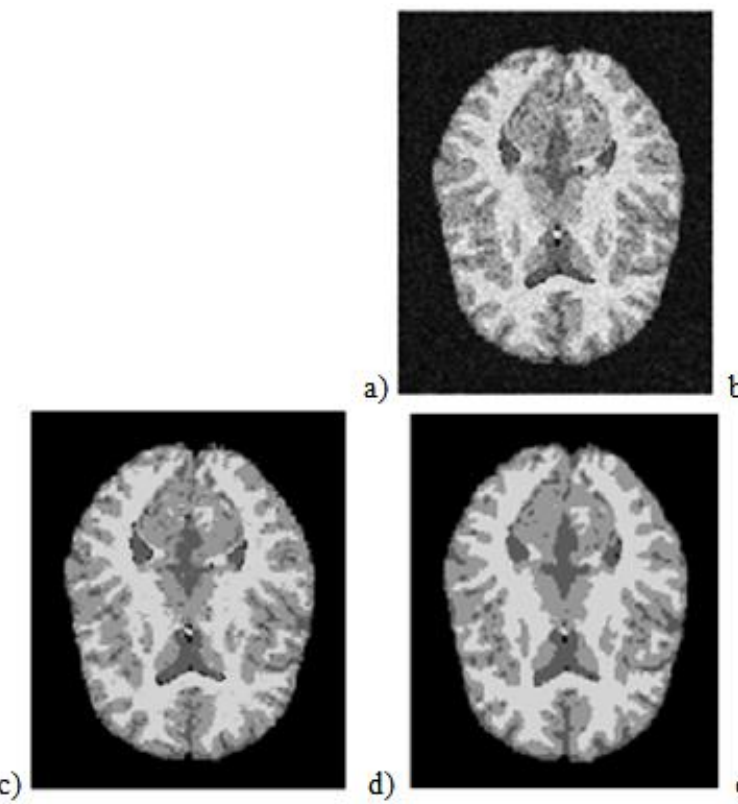
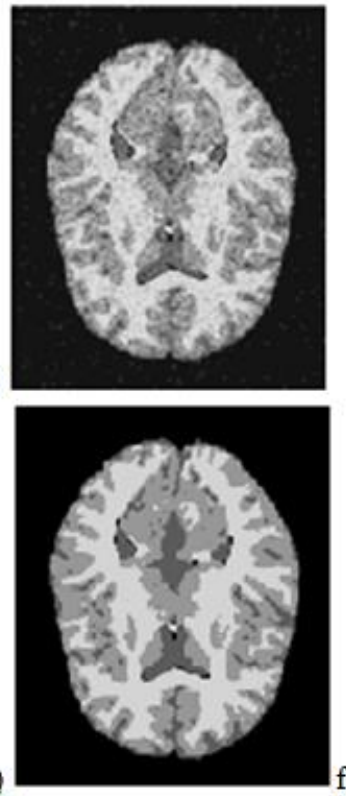

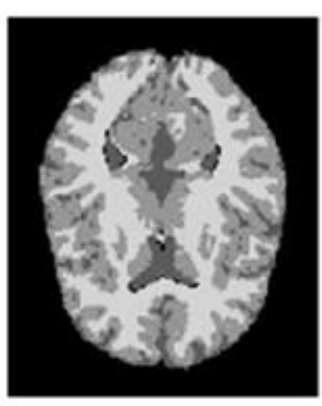

Fig.1 Segmentation of Simulated MRI brain image (a) Original image corrupted with 9\% Rician noise and segmentation results obtained by using b) FCM (c) Modified FCM (d) WIPFCM (e) FCM_NLS (f) Proposed algorithm

Table 1 Comparison of Segmentation accuracies on the Brain web T1-weighted images corrupted up to $9 \%$ Rician noise

\begin{tabular}{ccccccccccc}
\hline \multirow{2}{*}{ Algorithms } & \multicolumn{2}{c}{ FCM } & \multicolumn{3}{c}{ Modified FCM } & \multicolumn{2}{c}{ WIPFCM } & \multicolumn{2}{c}{ FCM_NLS } \\
\cline { 2 - 9 } & GM & WM & GM & WM & GM & WM & GM & WM & GM & WM \\
\hline $\mathbf{1 \%}$ & 98.28 & 98.53 & 97.32 & 98.20 & 97.04 & 97.71 & 97.11 & 98.08 & 97.66 \\
$\mathbf{3 \%}$ & 95.08 & 97.03 & 96.18 & 97.18 & 95.88 & 97.32 & 96.20 & 97.56 & 97.15 & 97.95 \\
$\mathbf{5 \%}$ & 93.38 & 93.22 & 95.89 & 96.98 & 95.25 & 96.48 & 95.40 & 96.86 & 96.23 & 97.35 \\
$\mathbf{7 \%}$ & 87.12 & 88.75 & 92.82 & 95.30 & 93.18 & 95.74 & 93.84 & 96.18 & 94.24 & 97.09 \\
$\mathbf{9 \%}$ & 81.63 & 84.14 & 90.58 & 94.22 & 91.11 & 94.80 & 92.15 & 95.01 & 92.56 & 95.47 \\
\hline
\end{tabular}

\section{Experimental Results and Discussion}

In this section, the proposed algorithm is implemented in MATLAB and experiments are performed on magnetic resonance (MR) images downloaded from the brain web simulated brain data base. The proposed algorithm is compared with the three other FCM extension algorithms, such as Modified FCM, WIPFCM and FCM_NLS. The performance of the image segmentation is measured quantitatively by the by using three indices, similarity index, false positive ratio $r f p$ and false negative ratio $r f n$. For a given image if $A k$ and $B k$ represents the sets of pixels belong to class $k$ in ground truth and in the segmentation result, then the similarity index for the matching pixel between $A_{k}$ and $B_{k}$ is defined as:

$\rho=\frac{2\left|A_{k} \cap B_{k}\right|}{\left|A_{k}\right|+\left|B_{k}\right|}$

Where $|$.$| is the total number of pixels. The false$ positive ratio $r_{f p}$ is error resulting due to misclassification in class $\mathrm{k}$ and the false negative ratio is the error due to loss of the desired pixels of class $k$ and they are given as: $r_{f p}=\frac{\left|B_{k}\right|-\left|A_{k} \cap B_{k}\right|}{\left|A_{k}\right|}$
$r_{f n}=\frac{\left|A_{k}\right|-\left|A_{k} \cap B_{k}\right|}{\left|A_{k}\right|}$

\section{A. Experiment on Simulated Brain MRI}

In this section, we evaluate the performance of the proposed algorithm on the simulated MRI Brain images obtained from the Brain web simulated data base [20] , a labeled T1 - weighted Brain MRI sequence having slice thickness of $1 \mathrm{~mm}$ and volume size $217 \times 217 \times$ 181 is downloaded from Brain Web. The image is a slice of simulated 3D volume, having size $217 \times$ 181.Before applying any segmentation algorithm to the simulated Brain MRI image, the extracranial tissues like skin, skull, glial matter, connective and blood vessels are removed and set as background pixel. The image to be processed includes only four classes, which are cerebrospinal fluid (CSF), grey matter (GM), white matter (WM) and the background. The image also acts as the ground truth for the segmentation process. MRI images are generally contaminated with Rician noise. Therefore, we add Rician noise into the image. Fig. 1b-f show the results of segmentation algorithms on simulated Brain MRI Image corrupted with 9\% of Rician noise. 


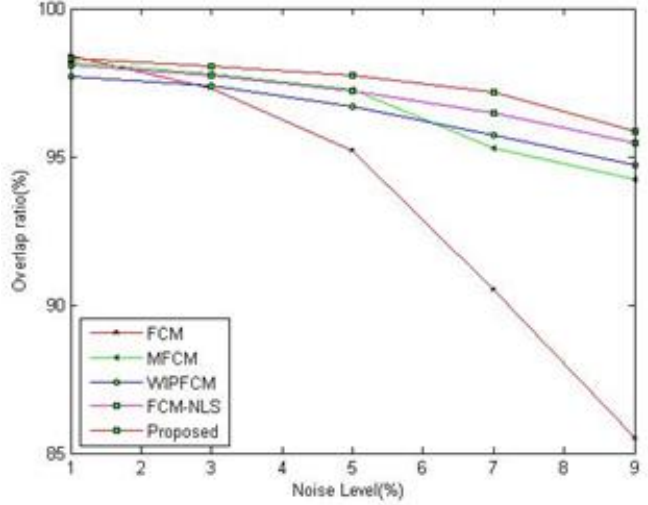

(a) WM

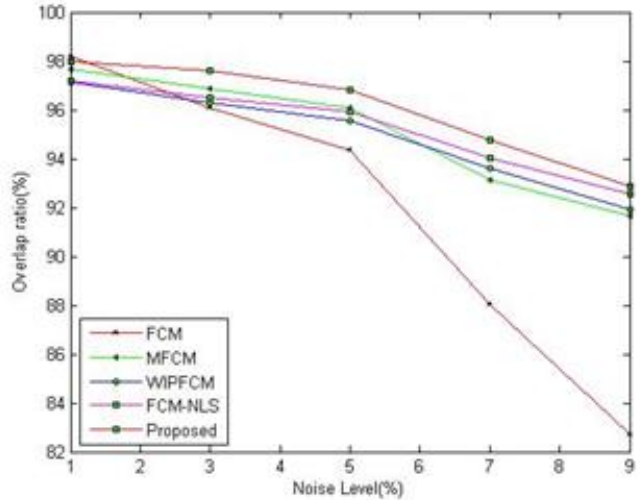

(b) GM

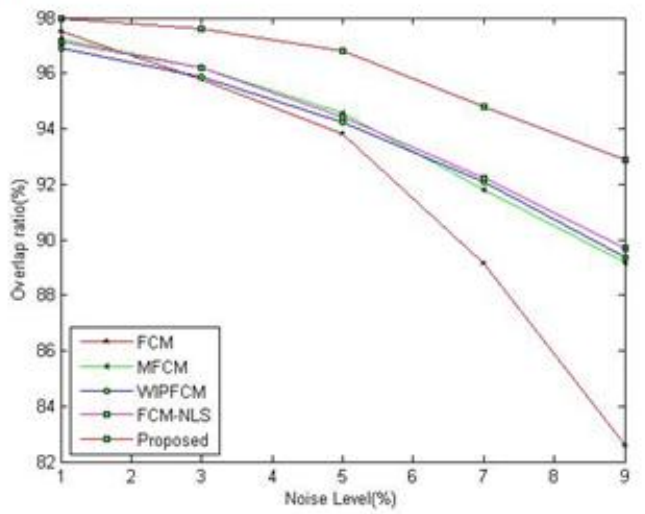

(c) CSF

Fig.2 Comparison of overlap ratios of different segmentation techniques on Brain web T1-weighted images with different Rician noise levels

From the results we can see that the FCM algorithm is not able to remove the noise, which results into false segmentation. On the other hand, modified FCM gives better results compared to traditional FCM algorithm, but still the noise can be seen in the segmented image, resulting in poor accuracy of the segmentation. The results obtained by WIPFCM and FCM_NLS are better than the Modified FCM algorithm, but still smoothness exists between the boundaries of the tissues. Therefore, the structural information present in the image is lost, but the results obtained by the proposed clustering algorithm are robust to noise and also it preserves the details and fine structures present in the image. To quantitatively evaluate the performance of all the algorithms, they are applied to the two dimensional images (Slice no.75, 80, 85, 90, $95,100,105,110,115$ and 120) affected by different noise levels $(1 \%, 3 \%, 5 \%, 7 \%$, and $9 \%)$. Here we set the neighborhood window size to $3 \times 3$ for all algorithms and weset the search region $\phi_{i}=7 \times 7$ for the Modified FCM and the proposed method, $\lambda_{g}=0.25$ for FCM_NLS and filtering parameter $\mathrm{h}=500$ for Modified FCM , the smoothing parameter $p=0.7$ using trial and error method for the proposed method. The quantitative evaluation in terms of overlap ratios for all the algorithms is shown in Table 1 . We can observe that the modified FCM algorithm works well for low noise levels and the performance of WIPFCM and
FCM_NLS is better in case of high noise. From the Table, it can be observed that overlap ratio for the proposed method is higher and it performs better in comparison to all the other algorithms in presence of both low and high noise levels. Fig. 2 shows the graphical comparison of the overlap ratios of each tissue with respect to noise for all the algorithms. These graphs show that the performance of the FCM algorithm decreases with the increase in the noise, while modified FCM performs better than FCM_NLS and the WIPFCM methods for noise level up to $5 \%$, but the performance of FCM_NLS and WIPFCM increases with the increase in percentage of noise. However, the proposed method show better results for both low and high noise levels.

\section{Conclusion}

In the Proposed algorithm, a technique to determine the filtering parameter ' $h$ ' is proposed. In the proposed method the filtering parameter ' $h$ ' in the non-local spatial information is directly calculated from the noise present in the image, making it adaptive with respect to the percentage of noise, which adaptively influence the similarity measure in the objective function, thereby and making it robust in presence of noise and preserving the details of the image. The performance of the proposed algorithm is tested on simulated brain 
MRI images from Brain Web data base under different percentage of noise levels. By quantitatively inspecting the experimental results we can conclude that the performance of the proposed algorithm is higher than FCM, Modified FCM, and WIPFCM and FCM_NLS algorithm. Our future work includes adaptively determining the size of search region and the smoothing parameter from the amount of noise present in the image.

\section{References}

J. Dunn (1974), A fuzzy relative of the ISODATA process and its use in detecting compact well separated clusters, Journal of Cybernet., vol. 3, pp. 32-57

Buades. A, Coll. B, Morel .J.M. (2005) A non-local algorithm for image denoising, In CVPR: 60-5

Bezdek. J.C (1981), Patter recognition with fuzzy objective function algorithm, New York: Plenum Press

Krinidis. S, Chatzis. V (2010), A robust fuzzy local information $\mathrm{C}$-means clustering algorithm, IEEE Trans. Image Process. 19, (5), pp. 1328-1337

D. Pham (1999), An adaptive fuzzy C-means algorithm for image segmentation in the presence of intensity inhomogeneities, Pattern Recognit. Lett., 20, pp. 57-68, 1999.

A, Coll. B, Morel J.M. (2004) ON Image denoising methods Technical Report, CMLA

L. Zhu, F. Chung ,S. Wang (2009), Generalized fuzzy C-means clustering algorithms with improved fuzzy partitions, IEEE Trans. Syst., Man, Cybern., B, Cybern., vol. 39, no. 3, pp. 578-591

X. Yin, S. Chen, E. Hu, and D. Zhang (2010), Semi-supervised clustering with metric learning: An adaptive kernel method, Pattern Recognit., vol. 43, no. 4, pp. 1320-1333

Zhao, F., Jiao, L. C., \& Liu, H. Q. (2011) Fuzzy c-means clustering with non-local spatial information for noisy image segmentation. Frontiers of Computer Science in China, 5, 45-56
L. Szilagyi, Z. Benyo, S. Szilagyii, H. Adam (2003), MR brain image segmentation using an enhanced fuzzy C-means algorithm, in Proc. 25th Annu. Int. Conf. IEEE EMBS, pp. $17-21$

W. Cai, S. Chen, D. Zhang (2007), Fast and robust fuzzy cmeans clustering algorithms incorporating local information for image segmentation, Pattern Recognit., vol. 40, no. 3, pp. 825-838

Aja-Fernandez. S, Westin. CF (2008), Noise and Signal estimation in magnitude MRI and Rician distributed images, a LMMSE approach, IEEE Trans.Image Process., $17(8), 1383-1398$

M. Mathworks and Natick, Image Processing Toolbox [Online]. Available: http://www.mathworks.com.

S. Chen, D. Zhang (2004), Robust image segmentation using FCM with spatial constraints based on new kernel-induced distance measure, IEEE Trans. Syst., Man, Cybern., vol. 34, pp. 1907-1916

J. Wang, J. Kong, Y. Lu, M. Qi, B. Zhang (2008), A modified FCM algorithm for MRI brain image segmentation using both local and non-local spatial constraints, Computerized Medical Imaging and Graphics, 32(8): 685-698

Zexuan. Ji, Y. Xia, Q. Chen, Q. Sun, D. Xia, D. Feng (2012) Fuzzy c-means clustering with weighted image patch for image segmentation, Applied Soft Computing, 12, 1659. 1667. 2012.

Z. Zaixin, C .Lizhi, C. Guangquan (2014), Neighbourhood weighted fuzzy c means clustering algorithm for image segmentation, IET Image processing, Vol. 8, Iss. 3, pp. 150 161

S. Chen and D. Zhang (2004), Robust image segmentation using FCM with spatial constraints based on new kernelinduced distance measure, IEEE Trans. Syst., Man, Cybern., B, Cybern., vol. 34, no. 4, pp. 1907-1916

Mathworks and Natick, Image Processing Toolbox [Online]. Available:http:// matlabcentral/fileexchange/14237.

Available: http://www.bic.mni.mcgill.ca/brainweb.

Available: http://www.cma.mgh.harvard.edu/ibsr/. 